\title{
NIEVES, HIELOS Y AGUAS EN LOS PAISAJES DE SIERRA NEVADA. EL INTERÉS DE LA INFORMACIÓN GRÁFICA DE LOS LIBROS DE ÉPOCA ${ }^{1}$
}

\author{
A. GÓMEZ ORTIZ, B. MILHEIRO SANTOS \\ y D. SERRANO GINÉ
}

Servei de Gestió i Evolució del Paisatge

Universitat de Barcelona

Correo electrónico de contacto: gomez@ub.edu

\begin{abstract}
RESUMEN. La documentación gráfica - a partir del siglo XVI (gráficos, dibujos, esquemas y mapas) - de los libros de época resulta valiosa en la interpretación geográfica del paisaje de Sierra Nevada. Y lo relativo a aguas, nieves e hielos resulta fundamental en la reconstrucción y explicación científica de la Pequeña Edad del Hielo. Las ideas e interpretaciones que se incluyen en este artículo (siglos XVII-XIX) ponen de relieve el progreso paulatino de la cartografía como documento geográfico y, al tiempo, resaltan el avance del conocimiento científico del paisaje de cumbres de Sierra Nevada.
\end{abstract}

ABSTRACT. The graphical documentation from ancient books is valuable in the landscape's geographical interpretation of Spanish Sierra Nevada. Water, snow and ice are fundamental in the reconstruction and scientific explanation of the Little Ice Age. The ideas and interpretations that are included in the paper (centuries XVII-XIX) stress the gradual progress of the cartography and emphasize the advance of the scientific knowledge of Sierra Nevada summit landscape.

Palabras clave: cartografía, documentos de época, hielos, información gráfica, Pequeña Edad del Hielo, Sierra Nevada.

Key words: cartography, ancient documents, ice, graphical information, Little Ice Age, Sierra Nevada

Enviado el 14 de julio de 2008 Aceptado el 2 de septiembre de 2008

1. Un resumen del presente artículo fue defendido en formato de comunicación oral en el Segon Congrès Català de Geografia, organizado por la Societat Catalana de Geografia (Barcelona/Vilanova i la Geltrú, 2008). 


\section{Introducción}

El paisaje de Sierra Nevada comenzó a interesar a estudiosos y científicos, principalmente, a partir de la información de los viajeros ilustrados. Hasta entonces el conocimiento que se tenía de la Sierra, en particular de sus tramos más elevados, procedía de relatos o crónicas de autores árabes donde se resaltaban, sobre todo, los pasos o collados que cruzaban las cumbres, la abundancia de nieves y aguas, así como la riqueza de plantas medicinales (Torres Palomo, 1967-1968). Fue a partir de las primeras décadas del siglo XVIII cuando se inicia su descubrimiento y representación gráfica, favorecido todo ello por las ideas de la Ilustración. Este cambio de actitud significó que viajeros y eruditos comisionados por la corona borbónica o la nobleza se adentrarán en la Sierra recopilando datos de su medio biofísico y, al tiempo, levantaran una incipiente cartografía, pues hasta entonces Sierra Nevada se representaba como un promontorio bien individualizado en los mapas de conjunto de la Península Ibérica o del Mediterráneo Occidental (Titos Martínez y Piñar Samos, 1995) (Fig. 1).

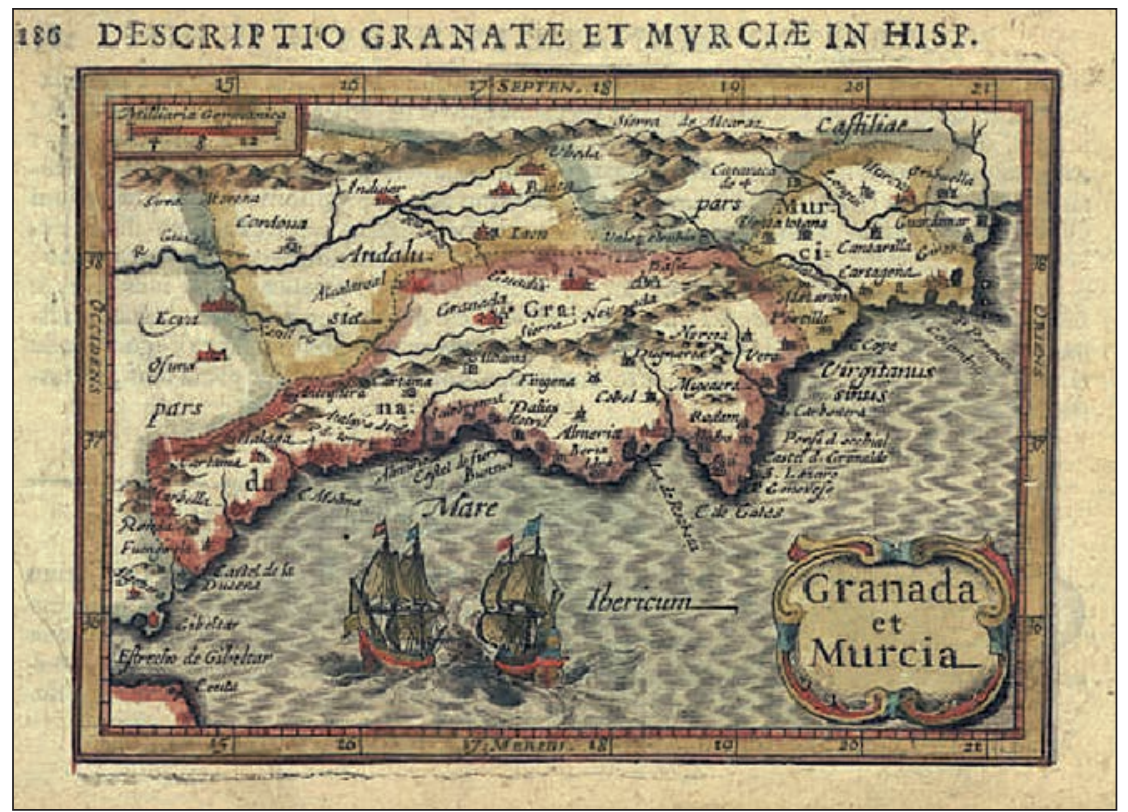

Figura 1. Sierra Nevada en el Reino de Granada (Bertius \&Honduis, 1616) 


\section{El marco geográfico}

Sierra Nevada es un macizo montañoso bien delimitado en el sureste peninsular. Alberga las mayores altitudes de la península Ibérica (Mulhacén, 3482 m; Veleta, 3394 m; Alcazaba, $3371 \mathrm{~m}$ ) y se dispone orientado de este a oeste, a lo largo de más de $90 \mathrm{~km}$, desde el surco del río Nacimiento hasta la vega de Granada. La Sierra fue poblada y aprovechados sus recursos naturales prontamente, tal como refleja la proliferación de núcleos de población dispersados por sus laderas y la marcada huella agraria (acequias, bancales y cortijillos) que remonta por encima de los 2000 m (Fig. 2).

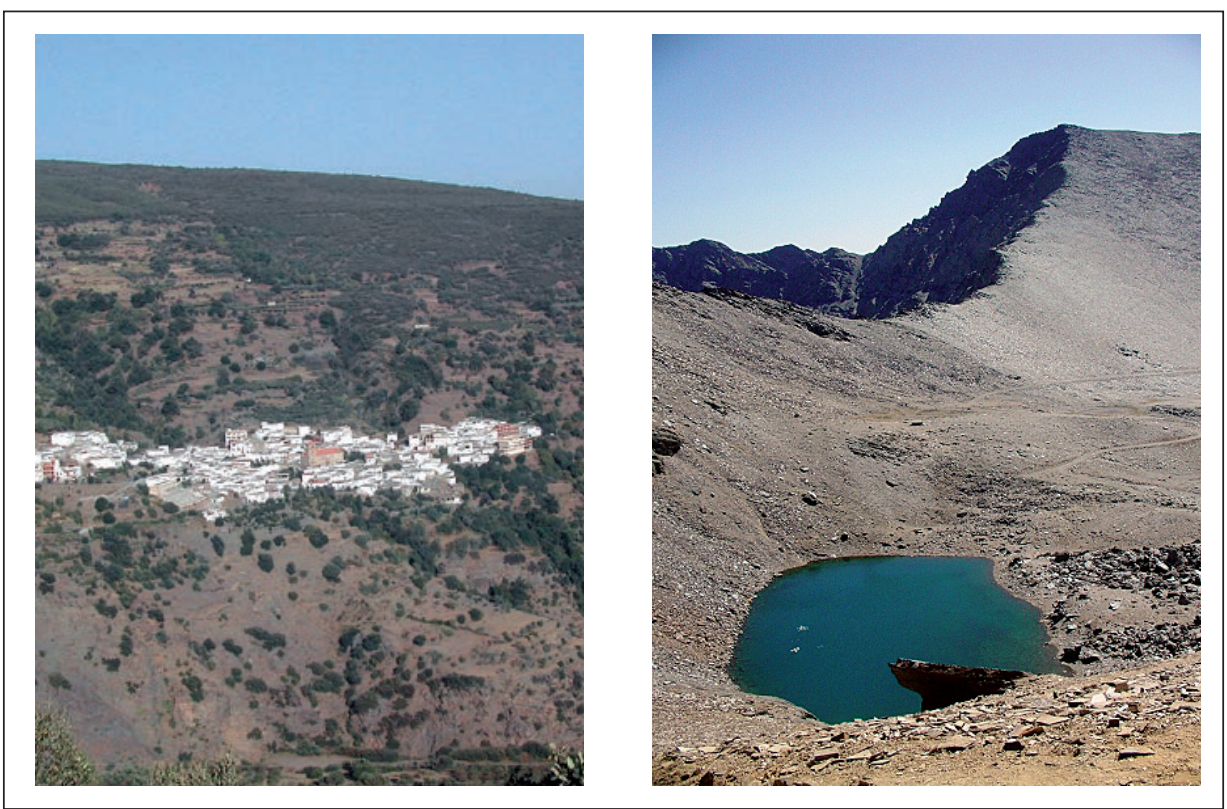

Figura 2. Bayarcal, en la Alpujarra almeriense, y laguna de la Caldera y cerro del Mulhacén (3482 m)

Respecto a los tramos de cumbres de Sierra Nevada hay que subrayar que resultan singulares con relación al conjunto de montañas andaluzas, en particular por su paisaje, definido por la huella glaciar, la presencia de nieves y la rica flora que alberga. Sin embargo, esta parte de montaña resultó secularmente territorio ignorado -sobre todo por imperativo de sus condiciones climáticas- sólo frecuentado en verano por pastores y recolectores de plantas medicinales. En la actualidad, las cumbres de Sierra Nevada están catalogadas como Reserva de la Biosfera, Parque Natural y Parque Nacional. 


\section{Objetivos, materiales y metodología}

El objetivo principal de este artículo es valorar el significado y alcance científico de la documentación gráfica de época en los escritos que describen el paisaje de cumbres de Sierra Nevada, particularmente, el medio biofísico. Y, de éste, interesa mayoritariamente lo relativo a aguas, nieves e hielos como indicadores de condiciones climáticas frías de la Pequeña Edad del Hielo. El periodo estudiado ha cubierto los siglos XVII, XVIII y XIX.

Los materiales analizados se centran en documentos escritos y cartografía (Tabla 1). Ésta, casi siempre, inmersa en libros, relatos o informes que describen particularidades del territorio de Sierra Nevada. La autoría de estos materiales es diversa, aunque por lo general se trata de personas eruditas y científicos. Primero, -a lo largo de finales del siglo XVII y todo el XVIII- ilustrados y, luego, -prácticamente todo el siglo XIX- prerománticos y románticos. Y en un caso y en otro con participación extranjera, casi siempre del centro de Europa.

Tabla 1. Relación de documentos analizados con indicación de fecha, autor y título

\begin{tabular}{|c|c|c|}
\hline Año & Autor & Título \\
\hline 1638 & Francisco Bermúdez de Pedraza & Historia eclesiástica de Granada \\
\hline $1640-46$ & Francisco Henríquez de Jorquera & Anales de Granada \\
\hline 1732 & Francisco Fernández Navarrete & Cielo y suelo granadino \\
\hline 1752 & Pedro Murillo Velarde & Geographia Histórica \\
\hline 1754 & Antonio Ponz & $\begin{array}{l}\text { Relación del viaje que desde } \\
\text { Granada hizo a Sierra Nevada }\end{array}$ \\
\hline 1795 & Tomás López y Vargas Machuca & Diccionario Geográfico \\
\hline 1804 & Simón de Rojas Clemente y Rubio & $\begin{array}{l}\text { Historia Natural del Reino de } \\
\text { Granada }\end{array}$ \\
\hline 1845 & Charles Edmon Boissier & $\begin{array}{l}\text { Voyage botanique dans le midi de } \\
\text { l'Espagne pendant l'anné } 1837\end{array}$ \\
\hline 1882 & Moritz Willkomm & Las Sierras de Granada \\
\hline 1889 & Luis de Rute & La Sierra Nevada \\
\hline 1893 & Juan B. Bide & $\begin{array}{l}\text { Deuxième excursion dans la } \\
\text { Sierra Nevada }\end{array}$ \\
\hline 1899 & Johannes J. Rein & $\begin{array}{l}\text { Aportación al estudio de Sierra } \\
\text { Nevada }\end{array}$ \\
\hline
\end{tabular}


En cuanto al método de trabajo utilizado ha sido bibliográfico, preferentemente. Tras la identificación y selección de documentos (libros, artículos, reseñas, conferencias -originales o traducidos-) se ha sintetizado su contenido y clasificado la información más relevante por campos temáticos. Acerca de la documentación gráfica (dibujos, esquemas, croquis, mapas, etc.) se han escaneado y depurado las imágenes convenientemente para su reproducción. En ocasiones, además, los sectores identificados se han localizado en el ortofotomapa (Junta de Andalucía, 1990) y elaborado panorámicas tridimensionales. De todo ello se ha conformado un banco de datos. Una vez sistematizada toda la información útil y tras ser interpretada, se ha ordenado y jerarquizado de acuerdo con los objetivos propuestos.

\section{Resultados y comentarios}

Tras el análisis de las obras consultadas hay que resaltar que los resultados obtenidos son valiosos en lo referente al paisaje de cumbres de Sierra Nevada, aunque más en lo relativo a información escrita que gráfica. El contenido de ambas fuentes ha suministrado datos de interés en cuanto a:

- La organización y características de las grandes unidades de relieve de Sierra Nevada.

- El medio biofísico y las condiciones ambientales de los tramos cimeros de Sierra Nevada.

- La representación gráfica del paisaje y del territorio

\subsection{Organización y características de las grandes unidades de relieve de la Sierra}

Todos los autores analizados insisten en resaltar la considerable magnitud de Sierra Nevada, así como sus elevadas cotas y profusión de barrancos que la atraviesan. Ya los primeros autores árabes, como al-Idrisi y al-Zuhri (TorresPalomo, 19671968) insistieron en ello, aunque siempre proporcionando datos genéricos. Al-Idrisi, en el siglo XII, al referirse a su volumen y longitud la califica de cadena de montañas: "Esta cadena se extiende en el espacio de dos jornadas; su altura es muy considerable y las nieves la cubren perpetuamente (...) la parte de montañas que se extiende hacia el sur puede verse desde el mar a una distancia de cerca de 100 millas”. Y por lo que respecta a al-Zuhri, conviene resaltar: "De este monte salen veinticinco ríos de los que dieciocho van a desembocar al mar Romano (Mediterráneo) y siete al Guadalquivir. Nadie puede subir a esta montaña ni andar por ella, salvo en la época de calor (...). No tiene acceso esta montaña más que por tres lugares especiales y cuando se sube por alguno de ellos y se ha llegado a la cumbre, se divisan desde allí numerosas regiones". 
Sin embargo, habrá que esperar al siglo XVII para tener noticias más precisas de las regiones de cumbres de Sierra Nevada, aunque todavía inmersas en descripciones generales y que, en ocasiones, resultan ser adaptaciones más o menos afortunadas de autores árabes (Gómez Ortiz y Plana Castellví, 2006). Pero, a pesar de ello, por su interés geográfico y carácter novedoso en datos conviene resaltar la "Historia Eclesiástica de Granada" (Bermúdez de Pedraza, 1638) y los "Anales de Granada" (Henríquez de Jorquera, 1640-1646), donde se refiere información geográfica de interés de la Alpujarra y de sus tahas. Por lo que respecta a las cumbres de la Sierra, sobresale de nuevo su considerable altitud, la dificultad de acceso y, sobre todo, la permanencia de la nieve y la frescura y abundancia de aguas, aparte del valor medicinal de sus plantas: "Está (se alude a Sierra Nevada) a cinco leguas de Granada y parece que está sobre sus casas, tanta es su altura; y lo nevado se extiende ocho leguas, desde el puerto de la Ragua, en el marquesado del Zenete, hasta Órgiva (...). Y no podía faltarle el agua en ella, si ha conservado la nieve que le envió el cielo después del Diluvio” (Bermúdez de Pedraza, 1638). El conocimiento más detallado del macizo de Sierra Nevada, y por lo que se refiere a la organización espacial de los principales rasgos morfohidrográficos y aquellos otros biofísicos, económicos y poblacionales, comenzó a definirse y a cartografiar en la segunda mitad del siglo XVIII, a partir de los croquis y esquemas realizados durante la elaboración del Catastro de Ensenada y, sobre todo, de los trabajos de Tomás López y Vargas Machuca (desde 1761) que pretendían el registro sistemático de toda la Península Ibérica, como comentaremos más adelante.

\subsection{El medio biofísico y condiciones ambientales de las cumbres de Sierra Nevada}

La información más importante sobre estas cuestiones comienza a aparecer a partir de mediados del siglo XVIII, que es cuando los distintos elementos naturales del paisaje de Sierra Nevada empiezan a ser tenidos en consideración como piezas clave del mismo. Las aguas, las plantas y los minerales ocupan siempre lugar destacado y también las condiciones climáticas, aunque desde el comportamiento que tienen las temperaturas, el viento, la nieve y el hielo. Y toda esta manera de proceder es como respuesta al impulso y progreso que experimentaron las ciencias al amparo de la Ilustración. Sin lugar a dudas, el autor de mayor relieve en estas décadas fue Simón de Rojas Clemente y Rubio, probablemente el primer científico - botánico- que se adentró y recorrió la Sierra con el deseo no sólo de describir aquello que le interesaba sino también de explicarlo en su contexto geográfíco, apoyándose en gráficos e ilustraciones de gran valor, como veremos oportunamente. Antes que él, sin embargo, otros ilustrados también se ocuparon de las cumbres de la Sierra informándonos de su paisaje, aunque escasamente explicándolo. En tal sentido, destacan tres autores: Francisco Fernández Navarrete, Pedro Murillo Velarde y Antonio Ponz (Fig. 3).

Del primero, resalta su obra "Cielo y suelo granadino" -publicada en 1734- en la que se relatan observaciones físicas, médicas y botánicas del territorio del arzobispado de Granada y, en particular, de la propia ciudad granadina y de Sierra Nevada, ofrecien- 


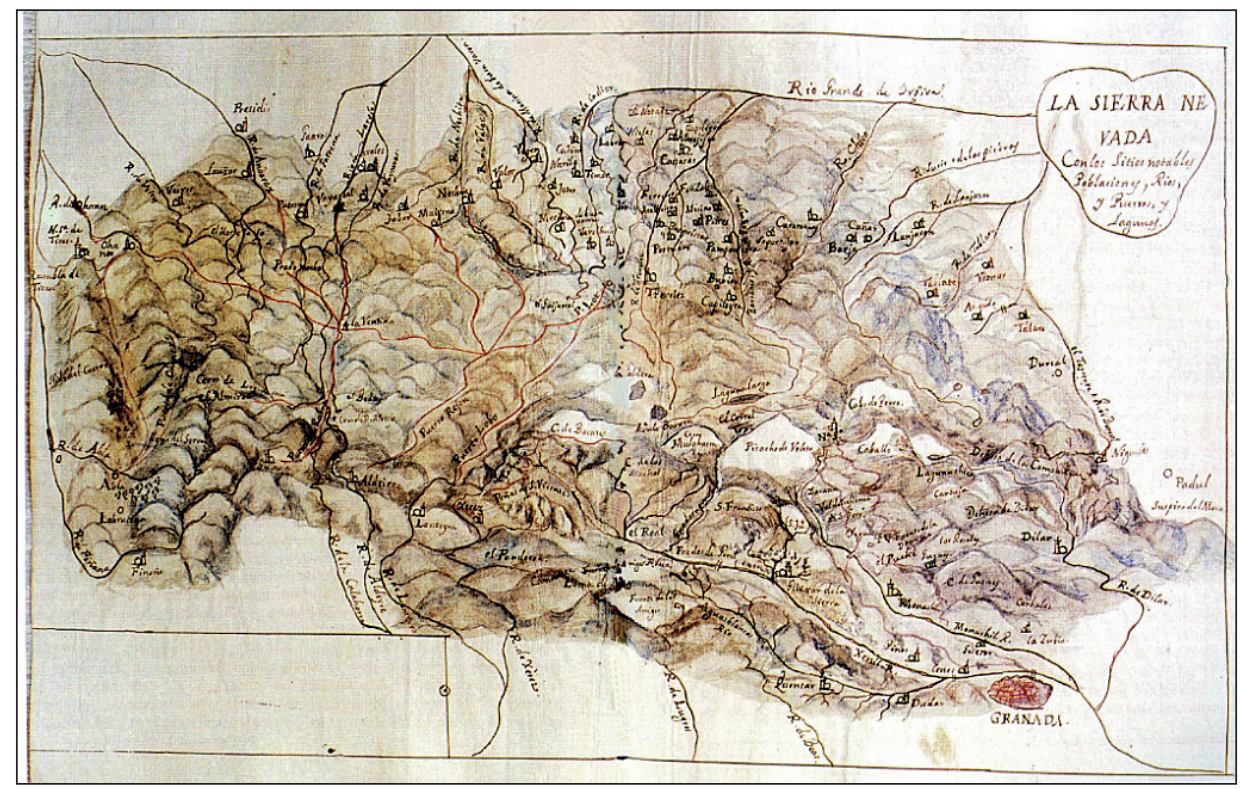

Figura 3. La Sierra Nevada, según Fernández Navarrete (1734)

do de esta última una amplia descripción y dos espléndidos dibujos panorámicos a color. Del segundo autor, subrayamos su "Geographia Histórica" (1752), en cuyo Tomo I, capítulo XIII ("Del Reino de Granada") ofrece información general de la ciudad de Granada y particular de las aguas y nieves de Sierra Nevada: "Allí (se refiere al corral y picacho del Veleta) hizo la naturaleza un pozo perpetuo, de donde se provee todo el año de nieve no solo una ciudad tan populosa, sino que se lleva de alli para otras partes de Andalucía sin que hay miedo de que jamás se acabe”. Y por lo que respecta a Antonio Ponz, quizá el primero que recorre la fachada norte y cumbres de la Sierra, allá por el año 1754, destacar la información que nos trasmite de su recorrido relativa a los neveros, lagunas y pastizales de las cabeceras de los barrancos. También de la panorámica que se divisa desde la cima del Picacho y, en particular, de las características del Corral del Veleta y de sus nieves “... caxon ambicioso de nieve, que se cree guarda la primera que cayó después del Diluvio, reducida a piedra, pues estando descubierto hacia el norte aqui es yelo lo que es nieve en otros lugares...".

Pero, como antes señalábamos, no hay dudas en afirmar que la mayor aportación durante estos tiempos se debió a Simón de Rojas Clemente y Rubio, botánico, discípulo de Antonio José Cavanilles, de quien asumió conocimientos y técnicas de estudio desde el Real Jardín Botánico de Madrid. Rojas Clemente recorrió la mayor parte de la Sierra, incluso sus cumbres -desde la Carihüela hasta el picón de Jeres-, describiendo y explicando el medio natural, que dejó escrito en su "Historia Natural del Reino de Granada". A pesar de que le preocupaba mayoritariamente la vegetación, que distribuyó en pisos altitudinales desde las orillas del Mediterráneo hasta las cimas del Mulhacén, 
Rojas Clemente hizo observaciones muy atinadas del relieve y de las condiciones ambientales en las que los vegetales se desarrollaban. Así, estableció que a partir de los 2436 m (2900 varas) los dos ambientes en los que las plantas crecían eran fríos, a los que calificó, al primero, de frigidísimo y, al contiguo y más elevado, glacial. De ambos remarcó la precariedad de vegetación que cubría el suelo y, sobre todo, insistió en la presencia de ventisqueros e hielos permanentes, así como la persistencia de los vientos que tendían a barrer la nieve de las altiplanicies: "Noto de paso que todas las altas cumbres de Sierra Nevada están peladas no porque deje de caer en ellas la nieve, sino porque la arrojan de ellas los vientos fuertes a que están expuestas ...” (Fig. 4).

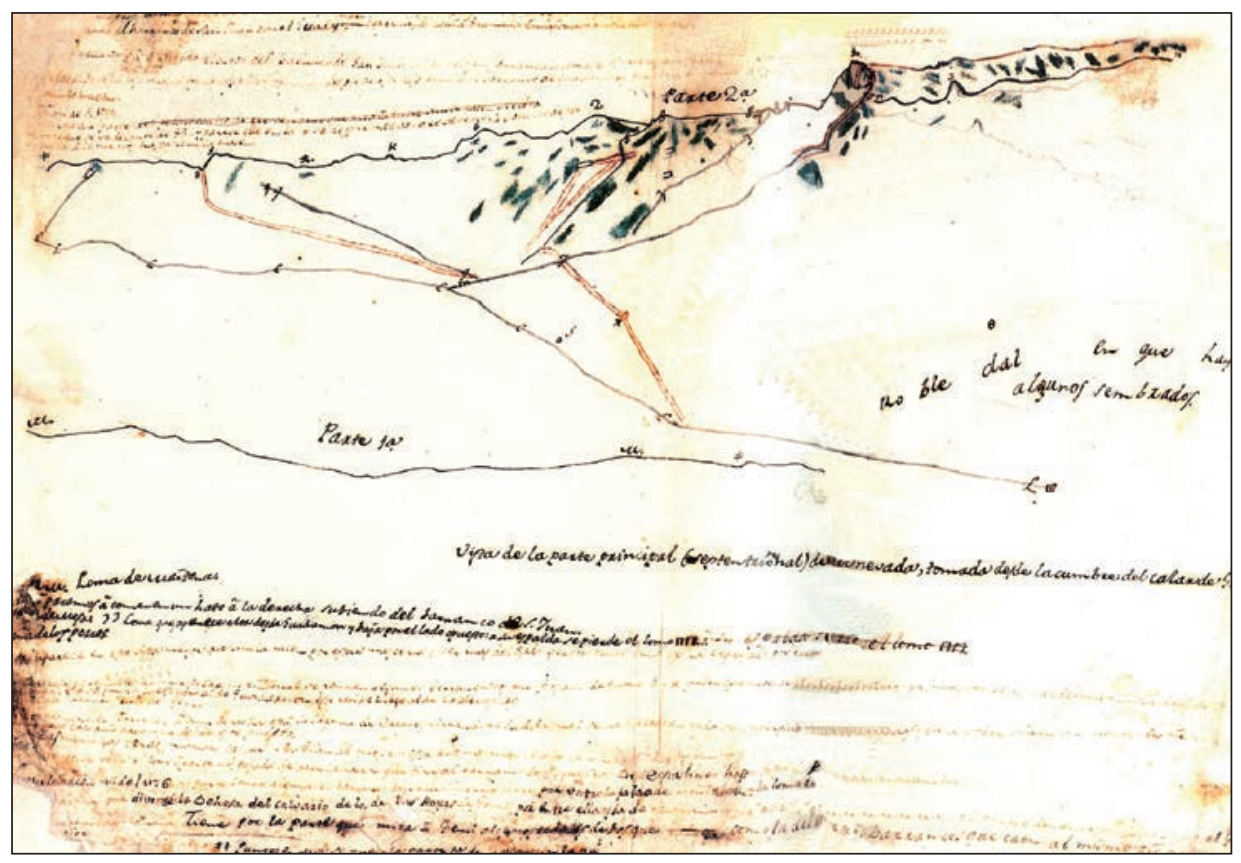

Figura 4. Neveros permanentes en fachada norte de Sierra Nevada (Rojas Clemente, 1804-1809)

Instalados en la mitad del siglo XIX Sierra Nevada es ahora recorrida por científicos naturalistas procedentes de universidades centroeuropeas. La Sierra, de nuevo, es foco de atracción por su rica flora y a su estudio se dedicaron, mayoritariamente, los ilustres visitantes que acuden a ella, entre los que destacaron Charles Edmon Boissier (1845), de la universidad de Ginebra, y Moritz Willkomm (1882), de las de Leipzig y Praga, ambos conocedores de los escritos Horace-Bénédict de Saussure y Ramond de Carbonnières, sobre los Alpes y Pirineos, respectivamente. De estos científicos, ahora conviene resaltar la información que suministraron de la geografía física de Sierra Nevada, en particular del relieve (líneas de cumbres -cotas, picos y pasos-, cabeceras 
de barrancos y lomas) y de las condiciones climáticas de las cumbres. Al respecto, Boissier, en su obra "Voyage botanique dans le midi de l'Espagne pendant l'année 1837", al relatar la herborización que realiza en el Corral del Veleta, califica por primera vez a los hielos en él atrapados como glaciares, describiendo y explicando, al tiempo, la configuración y morfología del cuenco: "Tiene la peculiaridad de ser el único en toda la Sierra y el más meridional de Europa: debe su formación a su posición, en el fondo de un circo abrigado y dominado en todas partes por altas cumbres donde las tormentas barren la nieve en invierno". Por su parte, también Willkomm en su libros de 1847 "Zwei Jahre in Spanien und Portugal" y 1882 "Aus den Hochgebirgen von Granada" nos aporta datos biofísicos de la Sierra, insistiendo en los mismos temas que Boissier, pues son los que identifican a las cumbres de esta montaña. En tal sentido, sólo destacar por nuestro interés particular, la atención que presta también al picacho del Veleta, como unidad de relieve singular y al que accedió en dos ocasiones: "Muy pegado al borde del vertiginoso abismo podía estudiar el colosal valle de circo prolongado al sur, en cuyo ángulo más al fondo, justo debajo de los despeñaderos gigantescos del Picacho, se veía la nítida silueta del helero del Veleta (...). Por encima del glaciar del Veleta se veía un enorme ventisquero aún más inclinado, que llega a una pared muy escabrosa, de una altura de casi 500 metros ..."

Durante los últimos decenios del siglo XIX la Sierra continuó siendo visitada por científicos y además, ahora, por excursionistas. Geólogos, botánicos y geógrafos resaltaron singularidades del paisaje de cumbres. Particularmente conviene señalar el interés por el reconocimiento de los terrenos geológicos (Drasche, 1878), sin duda, derivado de los trabajos iniciados por la Comisión del Mapa Geológico de España, con antecedentes en 1849. También se mostró atención al origen del relieve -la acción glaciar, sobre todo-, sobresaliendo en este caso Mac-Pherson (1875), que admitió abiertamente un glaciarismo cuaternario generalizado en las cabeceras de los barrancos de la Sierra, responsable de las lagunas. Pero tales ideas no fueron confirmadas definitivamente hasta comienzos del siglo XX con Otto Quelle (1908) y, particularmente, con Hugo Obermaier (1916), que cartografió, por primera vez, el dominio glaciado de la montaña, presentando, además, croquis de detalle, algunos a partir de fotografías.

Por lo que se refiere a la aportación de los excursionistas subrayar la distinta intencionalidad de los escritos. Los hubo que mostraron interés por la descripción de determinadas travesías -sobre todo aquellas que discurrían por los cordales de las altas cumbres-, descubriendo pasos entre laderas, determinando cotas, describiendo reparto de hielos y nieves en el suelo, etc. Tal fue el caso de Bide (1893), del Club Alpino Francés, en su artículo "Deuxième excursión dans la Sierra Nevada", donde se incluye un excelente esquema orográfico general del sector occidental del macizo, a escala 1/100000, en el que se identifica, por primera vez en la cartografía de la Sierra, el foco glaciar del Corral del Veleta.

Pero al tiempo que se desarrolla esta faceta excursionista "descubridora" de la montaña, se inicia otra de perfil pedagógico inspirada en los fines educativos de la Institución Libre de Enseñanza (1876) donde la montaña se interpretaba como motivo 
de estudio y de trabajo, resaltándose de ella sus valores paisajísticos y culturales. Y Sierra Nevada tampoco fue ajena a ello, pues en gran manera la obra de Luis de Rute (1889), "La Sierra Nevada", así viene a mostrarlo.

\subsection{Representación gráfica del paisaje y territorio}

La parte gráfica relativa a Sierra Nevada insertada en los documentos analizados (croquis, esquemas, dibujos, mapas, etc.), al menos hasta comienzos del siglo XVIII, resulta genérica. Hasta entonces la Sierra se representaba en su conjunto en mapas generales, aunque bien definida y delimitada (Titos Martínez y Piñar Samos, 1995). Probablemente, a partir del tercer decenio del siglo XVIII el reflejo gráfico del territorio comienza a formar parte de los escritos que se hacen de la Sierra. Sin embargo, se trata de una cartografía más imaginativa que real, aunque informa del territorio y de sus elementos. Para la primera mitad del siglo XVIII destacan las aportaciones que incluye Fernández Navarrete (1732) en su "Cielo y suelo granadino" (dos panorámicas de trazo artístico en color). Lo relevante de ellas es la distribución de la jerarquía fluvial -entre alineaciones de montañas, llanos y pequeños valles- y la fijación de las principales poblaciones. Una de las láminas, que incluye escala gráfica en leguas españolas y denomina "Demostración del Territorio de Granada", refleja la Vega de Granada, limitada en el horizonte por la pantalla de Sierra Nevada en la que resaltan dos elevaciones máximas (Veleta, que le asigna una altitud de 3 millas y Mulhacén). La otra lámina, del mismo estilo pictórico, recoge toda la demarcación del Arzobispado de Granada, vista desde el mar en dirección norte. En su conjunto goza de un gran valor geográfico pues muestra una visión particular pero bastante completa del territorio del arzobispado. De la información que ofrece, de nuevo resalta Sierra Nevada, con indicación de pasos, la vega de Granada, además de otras sierras (Gádor, Parapanda, etc.) y poblaciones. Y por lo que respecta a la red fluvial, como elemento estructurante del territorio, las cuencas del Genil, Guadalfeo, Andarax y Adra. También resalta la línea de costa en la que se distinguen torres de vigía, calas, albuferas y poblaciones (Fig. 5).

Pero la cartografía general del territorio de Sierra Nevada, como parte integrante de la general del "Mapa del Reyno de Granada" (1795), no empezó a aparecer hasta finales del siglo XVIII, al tiempo que se hacía también de toda la Península Ibérica. Resultó como fruto de los trabajos de recopilación geográfica llevados a cabo por Tomás López y Vargas Machuca a partir de las contestaciones al interrogatorio enviado a los alcaldes o párrocos de los pueblos, cuyos resultados también fueron utilizados por Pascual Madoz (1849) en su "Diccionario Geográfico-Estadístico-Histórico". En tal interrogatorio se solicitaban datos de los pueblos y sus demarcaciones, además de un pequeño mapa o croquis del término parroquial. El resultado fue la redacción del llamado "Diccionario Geográfico" (1799). Para el caso de Sierra Nevada la información recopilada fue relevante y variada sobre cuestiones geográficas, económicas, históricas, culturales, etc. -como refleja lo relativo a Güejar Sierra-, aunque no tanto su plasmación cartográfica. En tal sentido, la representación de Sierra Nevada refleja una falta de articulación inte- 


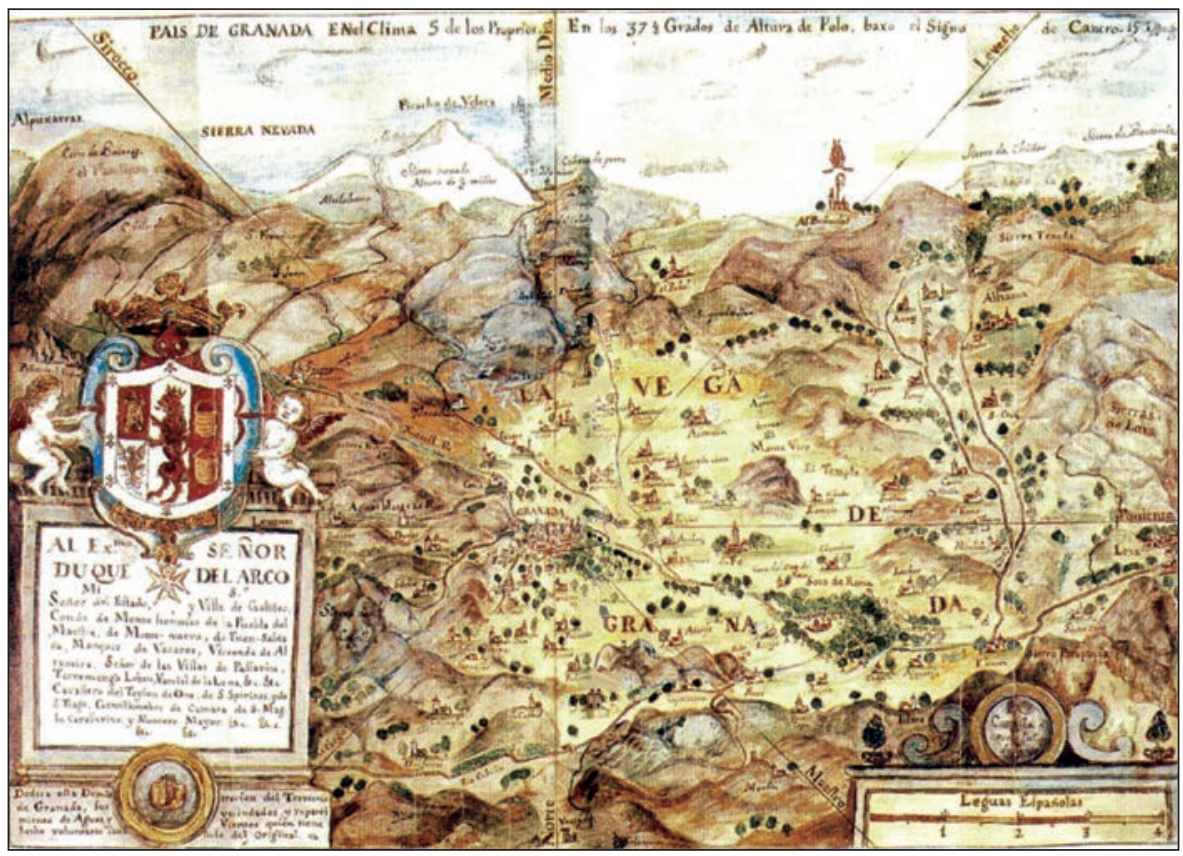

Figura 5. Panorámica de la vega de Granada y Sierra Nevada (Fernández Navarrete, 1732)

rior de la montaña, en particular en lo relativo a la distribución de ríos/valles y orientación de cuerdas montañosas, lo que propicia confusión en lo que respecta a la magnitud, altitudes del macizo y cabeceras de barrancos, lo que viene a mostrar el poco conocimiento que se tenía de las áreas de cumbres. A pesar de ello se logró componer una cartografía general aproximada de la Sierra, sobre todo en lo referente a organización de las redes fluviales principales, ubicación de las poblaciones y pasos entre vertientes de máximas cumbres (Fig. 6).

Instalados en el siglo XIX la presencia de naturalistas en la Sierra propició que sus paisajes comenzaran a interpretarse con rigor científico, lo que implicó que las obras editadas se enriquecieran con dibujos, gráficos, esquemas y mapas temáticos de gran interés. Así ya se observa en las aportaciones de Rojas Clemente y Boissier, botánicos ya referidos. Del primero, y formando parte de su excelente obra "Historia Natural del Reino de Granada", hay que resaltar los abundantes esquemas que aporta, todos relacionados con características geográficas del paisaje de la Sierra -en particular de orden botánico, con aportaciones morfológicas, litológicas y de usos agrarios-. Y por lo que respecta a la morfología y presencia de nieves en las cumbres de la Sierra interesa destacar dos esquemas relativos a los perfiles de los cordales cimeros y divisorias de aguas de su vertiente norte. Uno -realizado desde la cumbre del Veleta-, abarca desde el collado del cerro de los Machos hasta el puerto de Bacares (Fig. 4). El otro, desde el cuenco del Alhorí hasta el puerto del Lobo, cercano éste al de la Ragua. En ambos 


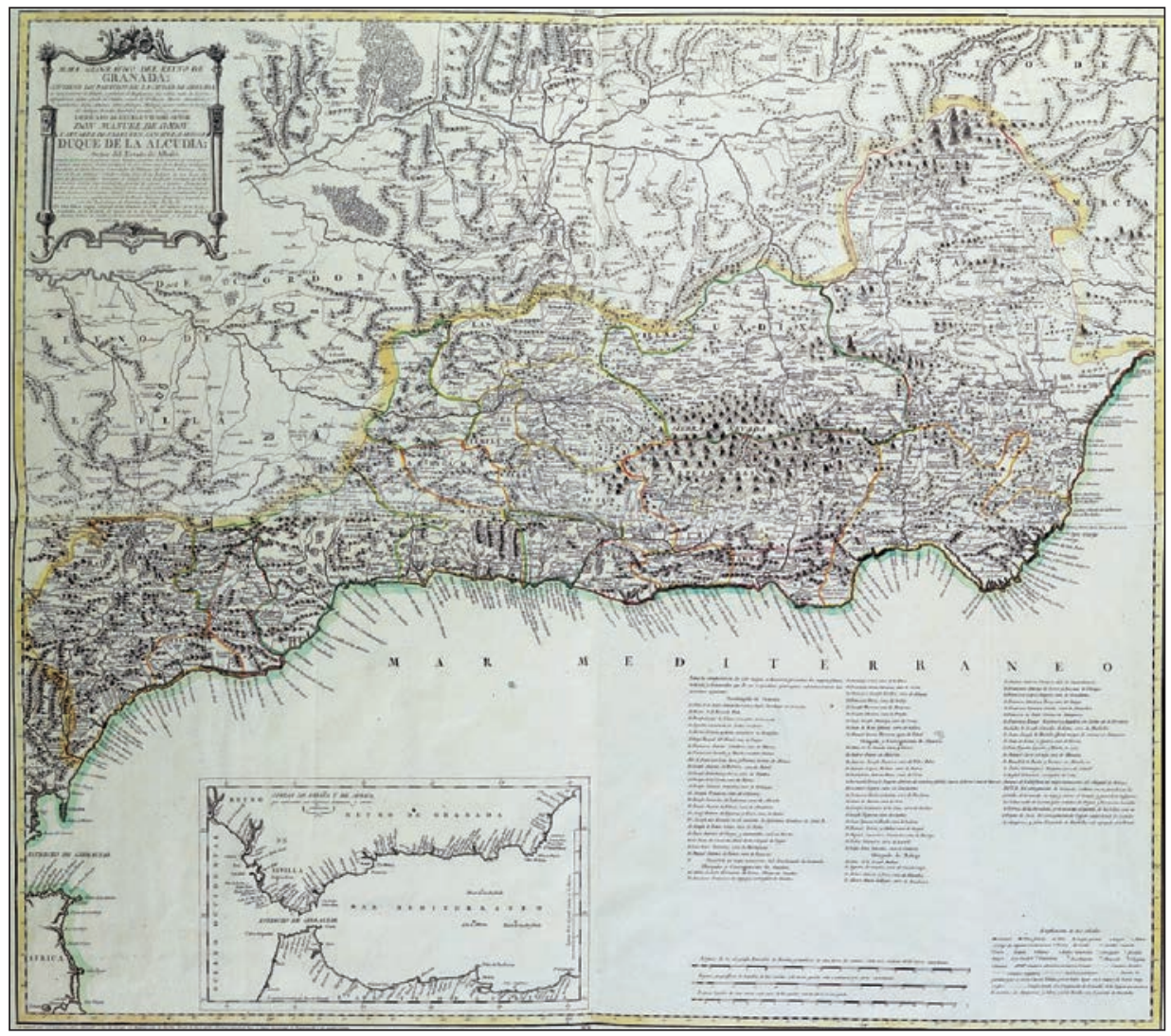

Figura 6. Cartografía general del Reino de Granada (López y Vargas Machuca, 1799)

esquemas Rojas Clemente incluye anotaciones relativas a la toponimia de picos, lomas y barrancos, y con mayor intencionalidad datos relativos a las manchas de nieve que distingue (ventisqueros permanentes y neveros de fusión tardía) que, además, resalta en trazos de color rojo, lo que permite apreciar el reparto del manto nival en las cimas de la Sierra en pleno mes de agosto de 1805 , que es cuando fueron llevadas a cabo las observaciones.

La aportación gráfica de Boissier, incluída en su libro ya citado "Voyage botanique dans le midi de l'Espagne pendant l'année 1837”, también posee un valor muy notable, en particular los dibujos artísticos de las plantas seleccionadas, plasmadas en láminas a todo color. Igualmente resulta de gran interés científico el esquema relativo al escalonamiento en altura de la vegetación de las montañas del Reino de Granada, quizá el primero de su tipo que incluye franjas bioclimáticas (zona cálida, zona montañosa, zona alpina y zona glacial), sierras y vegetales más característicos de ellas. Por lo que se refiere a las cumbres de Sierra Nevada, instaladas por encima de los 8000 pies (a partir de 
los Peñones de San Francisco), sobresalen las franjas alpina y glacial -las únicas en el conjunto de montañas andaluzas- caracterizadas por la presencia de nieves e hielos glaciares (glaciar del Corral del Veleta) (Fig. 7): "En los Alpes a esta altitud ya se hayan las nieves perpetuas, aquí nada parecido (...); sin embargo, la nieve caracteriza nuestra región, aunque solamente bajo la forma de manchas o montones acumulados en los bajos fondos y pliegues del terreno (...). Desde final de septiembre, toda la región se cubre de una nieve nueva, que sólo desaparecerá parcialmente a partir de junio, y la cubre pues completamente durante ocho meses".

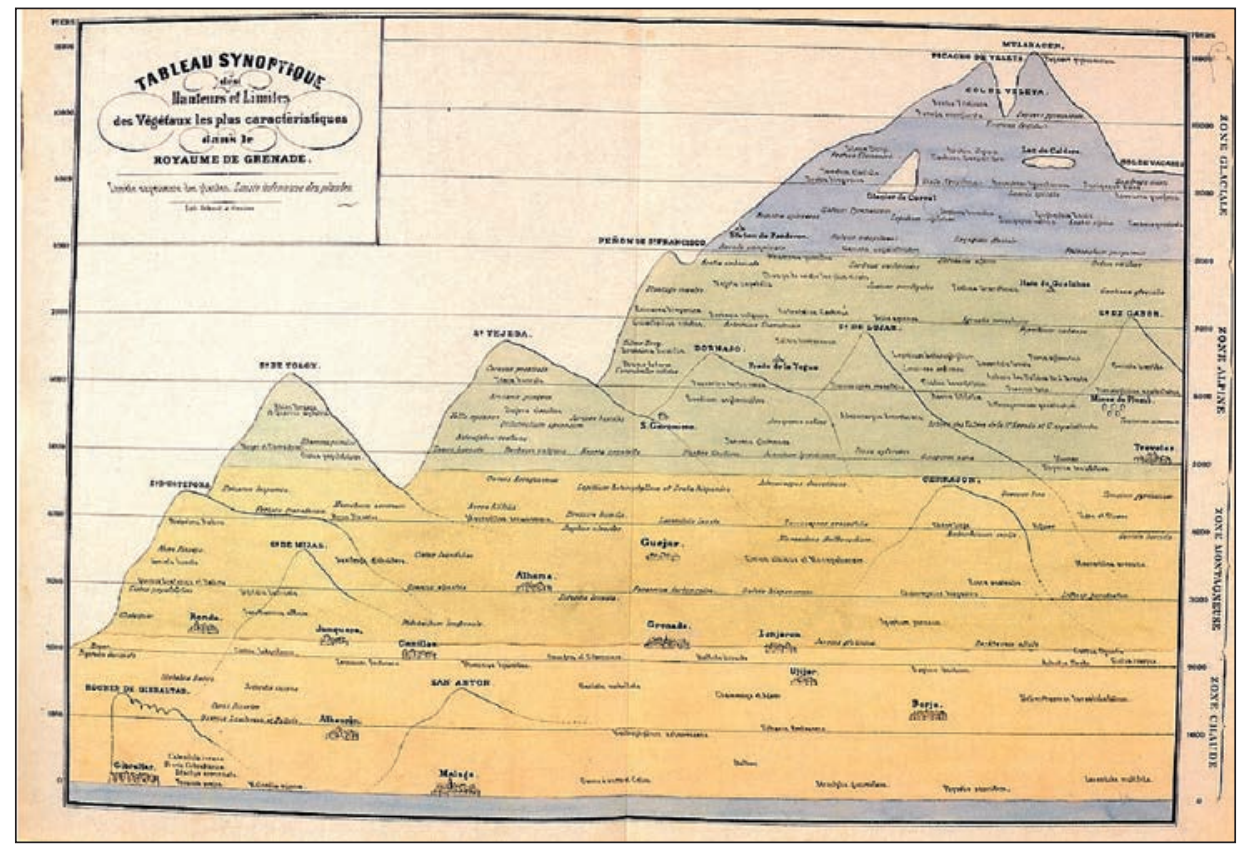

Figura 7. Pisos bioclimáticos propuestos por Boissier (1845).

La zona glacial es específica de Sierra Nevada

Décadas más tarde la información gráfica suministrada por los estudios sobre Sierra Nevada continuó incrementándose. En tal sentido destaca el mapa de los terrenos geológicos del macizo (escala 1/400000), publicado en el Boletín de la Comisión del Mapa Geológico de España (Drasche, 1879), dentro del programa de actuación iniciado por esta misma Comisión en 1849 (Fig. 8). El interés del mapa radica, ante todo, en la distribución espacial de las formaciones litológicas, destacando las rocas cristalinas y pizarras serpentínicas, que son las mayoritarias en las cumbres del macizo.

En otro orden de cosas también es de gran interés la cartografía de Bide (1893) que, aún siendo esquemática y a escala $1 / 100000$, resulta precisa por la técnica de sombreado utilizada y por el efecto óptico que produce la representación de los cordales y valles. 


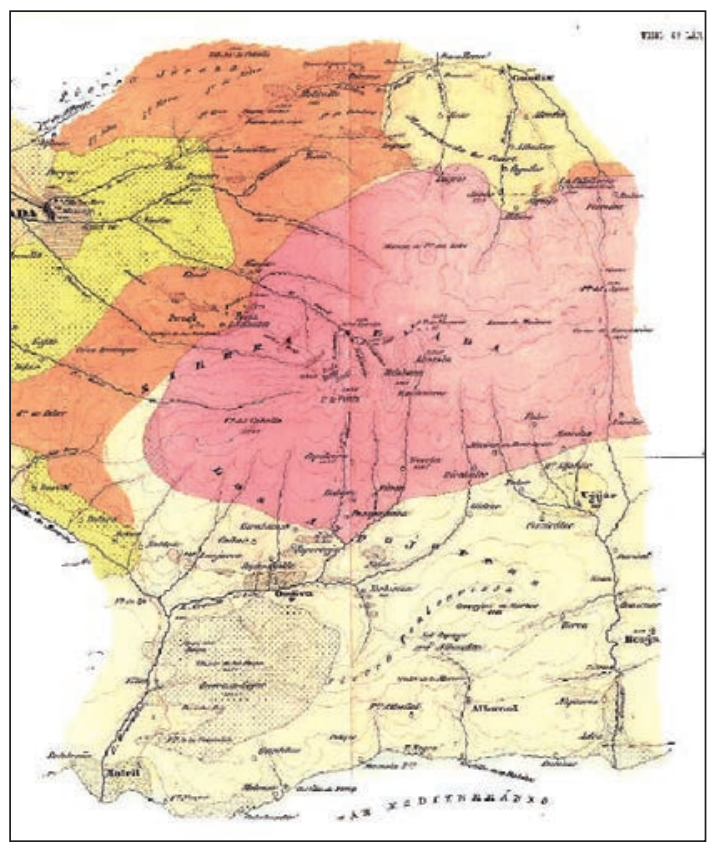

Figura 8. Fragmento del mapa geológico de una parte de Sierra Nevada (Drasche, 1878)

Además, y esto es lo más importante para valorar la dimensión del glaciarismo histórico en Sierra Nevada a finales del siglo XIX, refleja con suma precisión por vez primera y como se ha señalado, la localización del glaciar del Corral del Veleta, bien ajustado a la caja del circo del Guarnón (Fig. 9). También deben remarcarse diferentes gráficos panorámicos realizados a partir de fotografías, entre los que destacan las cabeceras de los barrancos de la fachada norte de la Sierra, desde el puerto de Bacares hasta los Lastrones del Veleta (Fig. 10).

Ya por último subrayar la cartografía general de Sierra Nevada que Rein (1899) incluye en su estudio "Beiträge zur Kenntnis der Spanischen Sierra Nevada". El mapa elaborado, a escala 1/200000, incluye la totalidad de la Sierra y regiones vecinas pudiéndose considerarse de los más completos de su tiempo. Incluye poblaciones, vías de comunicación, alineaciones montañosas representadas a través de sombreado y red fluvial. Fue diseñado y compuesto en el Instituto Geográfico-Militar de Viena, a partir de apuntes y bosquejos topográficos ya realizados por Francisco de Coello y Federico Botella, principalmente (Titos Martínez y Piñar Samos, 1995). El rigor de su información hizo que fuera documento cartográfico preferente durante muchos años. 


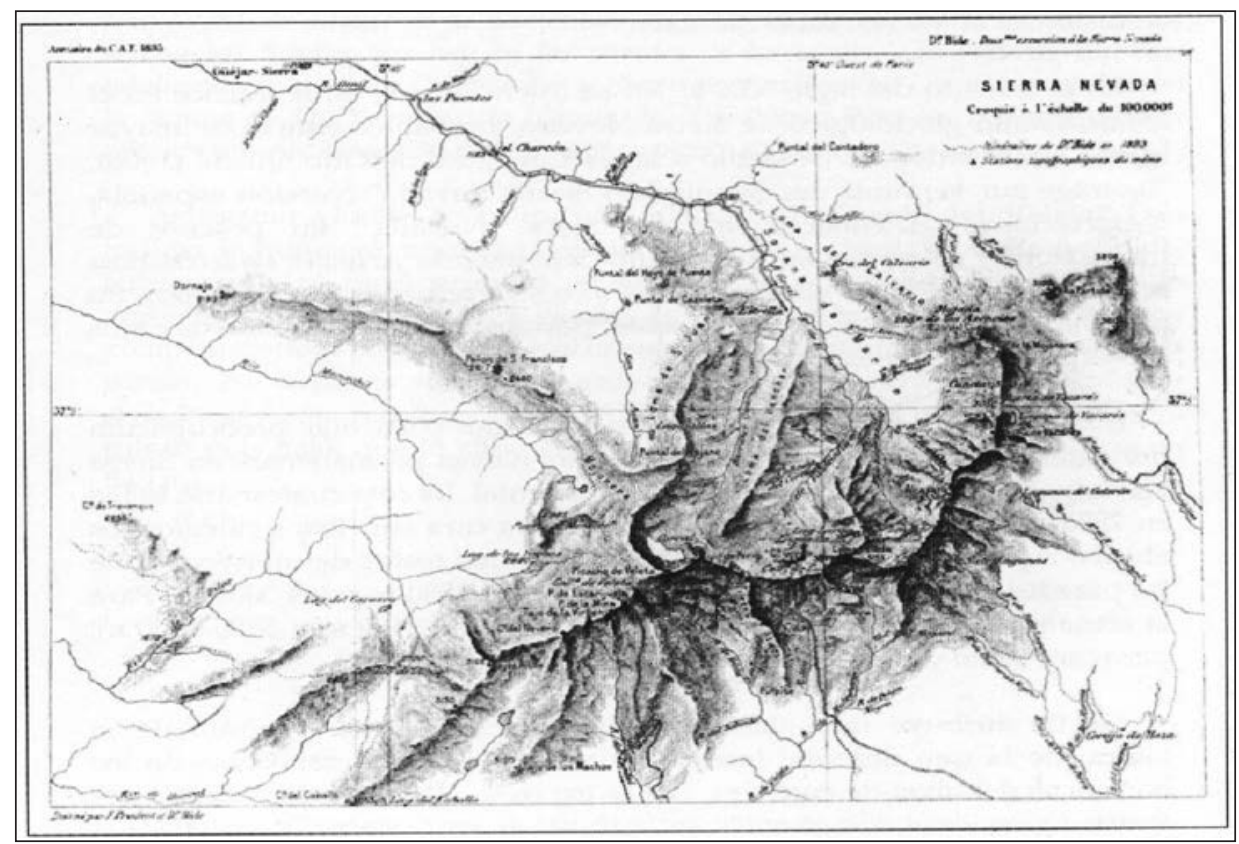

Figura 9. Croquis orográfico de las cumbres de Sierra Nevada y localización del glaciar del Veleta (Bide, 1893)

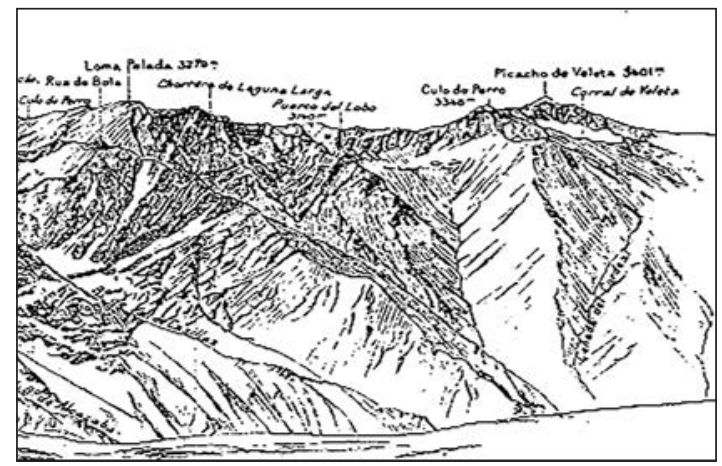

Figura 10. Croquis de la fachada norte de Sierra Nevada (Bide, 1893) 


\section{Conclusiones}

Las primeras cartografías de cierto detalle de Sierra Nevada proceden de autores ilustrados a quienes preocupó la organización del relieve, que lo articularon en torno a la red fluvial (resaltando picos, pasos, cotas y cursos fluviales) (Fernández Navarrete, 1743). También interesó la fijación de las poblaciones, el trazado de caminos y determinados usos del suelo. En su conjunto se trata de una cartografía más imaginativa que precisa pero de gran interés, pues distribuye en el territorio información geográfica relativa al paisaje (López y Vargas Machuca, 1795).

Entrado el siglo XIX, al amparo del progreso de las ciencias, las Naturales en particular, la información gráfica de época de Sierra Nevada comenzó a adquirir categoría de documento geográfico, al fijar en el espacio datos particulares del medio biofísico y, al tiempo, dar apoyo a las descripciones o explicaciones escritas, lo que significó la aparición de una cartografía temática (Rojas Clemente, 1804-1809; Boissier, 1845). En tal sentido y para el caso que nos ocupa, los datos relativos a la vegetación -organización altitudinal de especies-, al relieve y al clima -perfiles de cumbres, divisorias de aguas y distribución de hielos y nieves en el suelo- resultan relevantes.

Instalados en la segunda mitad del siglo XIX la cartografía de Sierra Nevada resulta ya bastante completa y precisa -aunque las alineaciones montañas todavía se representan en sombreados. Al respecto, la aportación de Rein (1899) es muy notoria. En cuanto a la denominada cartografía temática también alcanza durante este periodo notable expresión, como lo indica el bosquejo del Mapa Geológico de Sierra Nevada (Drasche, 1878) donde se resalta la distribución de los terrenos geológicos. Pero quizá la aportación más novedosa que conviene subrayar es la carta orográfica de Bide (1893) que refleja, por primera vez, la localización del glaciar del Corral del Veleta, que permite apreciar su extensión.

\section{Agradecimientos}

Al proyecto CICYT del Ministerio de Educación y Ciencia (Ref. SEG200500504/GEOG). También al de Parques Nacionales (Ref. 018/2007), del Ministerio de Medio Ambiente. Igualmente al Institut Cartogràfic de Catalunya y a Vicenç Casals por su colaboración en la parte gráfica.

\section{Referencias bibliográficas}

ABU-ABD-AlLA-MOHAMED-AL-IDRISI (1901). Descripción de España (versión española). Obra del siglo XII. Imprenta y Litografía del Depósito de la Guerra. Madrid. 
BermúdeZ de PedraZA, F. (1638). Historia eclesiástica de Granada. Edición facsimil. Universidad de Granada. Granada, 1989.

BIDE, J.B. (1893). Deuxième Excursion dans la Sierra Nevada. Annuaire du Club Alpin Français, Vol. XX: 276-305.

BOISSIER, C.E. (1845). Voyage botanique dans le midi de l'Espagne pendant l'anné 1837. Versión española en "Viaje botánico al sur de España durante el año 1837". Fundación Caja de Granada y Universidad de Málaga. Granada.

DRASCHE, R. (1878). Bosquejo geológico de la zona superior de Sierra Nevada. Boletín de la Comisión del Mapa Geológico de España. Tomo VII. Madrid.

Fernández Navarrete, F. (1732). Cielo y Suelo Granadino. Transcripción, edición, estudio e índices por A. Gil Albarracín. GBG. Editora. Almería-Barcelona, 1997.

Gómez Ortiz, A. y Plana Castellví, J.A. (2006). La Pequeña Edad del Hielo en Sierra Nevada a partir de los escritos de época (siglos XIII-XIX) y su relación con el progreso de la geografía física y geomorfología española. Boletín de la Asociación de Geógrafos Españoles, 42: 71-98.

HenríqueZ De Jorquera, F. (1640-1646). Anales de Granada. Reedición a cargo de Universidad de Granada-Ayuntamiento de Granada. Granada.

LÓPeZ y VARGas MachuCA, T. (1795). Diccionario Geográfico. En "Diccionario Geográfico de Andalucia: Granada”. Editorial Don Quijote. Granada, 1990.

MacPherson, J. (1875). De la existencia de fenómenos glaciares en el sur de Andalucía durante la época cuaternaria. Actas de la Sociedad Española de Historia Natural, Vol. IV: 235-246.

MADOZ, P. (1849). Diccionario Geográfico-Estadístico-Histórico de España y sus posesiones de ultramar. Madrid, Tomo XIV, pp. 383-386.

Murillo Velarde, P. (1752). Geografía Histórica. Edición del Reino de Granada (en Tomo I) en "Geografía de Andalucía”. Biblioteca de la Cultura Andaluza. Editoriales Andaluzas Unidas. Sevilla, 1988.

Obermaier, H. (1916). Los glaciares cuaternarios de Sierra Nevada. Trabajos del Museo Nacional de Ciencias Naturales (Geología), Vol. 17: 1-68.

PonZ, A. (1754). Relación del viaje que hizo desde Granada a Sierra Nevada D. Antonio Ponz a influxo del Excmo. Sr. Marqués de la Ensenada. Mensajero Económico y Erudito de Granada. Granada

Quelle, O. (1908). Beitragë zur Kenntnis der Spanischen Sierra Nevada. Tesis doctoral. Universidad Friedrich-Wilhelm. Berlin. 
REIN, J. (1899). Beitragë zur Kenntnis der Spanischen Sierra Nevada. Versión española en "Aportación al estudio de Sierra Nevada". Caja General de Ahorros de GranadaJunta de Andalucía. Granada.

Rojas Clemente y Rubio, S. (1804-1809). Historia Natural del Reino de Granada. En "Viaje a Andalucía. Historia Natural del Reino de Granada (1804-1809)", transcripción, edición, estudio e índices por A. Gil Albarracín. GBG. Editora. Barcelona, 2002.

Rute, L. (1889). La Sierra-Nevada. Imprimerie Charaire et fils. Paris.

Titos Martínez, M. y Piñar Samos, J. (1995). Album cartográfico de Sierra Nevada. 1606-1936. Fundación Caja de Granada. Granada.

TorRes PALOMO, M.P. (1967-1968). Sierra Nevada en los escritores árabes. Miscelánea de Estudios Árabes y Hebraicos. Vol. XVI-XVII: 57-88.

WiLlKomm, M. (1882). Aus den Hochgebirgen von Granada. Versión española en "Las Sierras de Granada". Caja General de Ahorros de Granada-Sierra Nevada 95. Granada. 